\title{
Applying Fusion of Pso-Abc Algorithm on the Minimax Location Problem
}

\author{
Jianfang Wang ${ }^{1}$, Ning Cheng ${ }^{1}$, Zhidu Liu ${ }^{1}$ and Changwang Liu ${ }^{2, *}$ \\ ${ }^{1}$ School of Computer and Information Technology, Nanyang Normal University, Nanyang, 473061, China; ${ }^{2}$ School of \\ Software, Nanyang Normal University, Nanyang, 473061, China
}

\begin{abstract}
In this paper, we propose a fusion of PSO-ABC algorithm based on the research of particle swarm optimization (PSO) algorithm and artificial bee colony (ABC). The former is apt to trap in local optimum, while the later has great ability of global searching and lower convergence speed in the later evolution process. Using the evolution of artificial swam, we gained a global optimum which was applied to the evolution of particle swarm to overcome the basic PSO's shortcomings of slow updating speed in later evolution process and that improved the local search ability of PSO algorithm within feasible region. This novel algorithm can solve the minimax location problem effectively and has a good application value.
\end{abstract}

Keywords: ABC, fusion algorithm, location problem, PSO.

\section{INTRODUCTION}

Minimax problem is a sort of non-differentiable optimization problem in mathematics. It can be used effectively to deal with many problems in real life, such as with least amount of work or doing most things in given time. Location problem is such a problem that can use minimax method to determine the optimization location site which will make the farthest site as much nearly as possible. For example, how to select the location site when establishing logistics distribution center, how to locating the place where the event took place to implement emergency rescue for emergency rescue facility when sudden event happens, all the above problems are in the domain of minimax problems, which can be solved with location algorithm[1]. A new algorithm is advanced to solve location problem based on careful study of minimax algorithm, that is, improved fusion of PSO and ABC.

\section{PSO AND ABC}

\subsection{PSO}

Suppose that in a D-dimensional target search space, there are $n$ particles which are the solutions of every optimum problem. Each particle has a fitness value determined by optimal function and a speed to determine their flying direction and distance [2]. Then particles will follow the current optimal particle to find their optimal solution in solution space iteratively. In each process of iteration, particles update themselves by tailing after two extremes. One is the optimal solution which is found by the particle itself, called pBest, the other, called gBest, is the optimal solution of the whole swarm. In t-th iteration, the current position of particle $i$ is expressed as follows: $x i(t)=(x i 1(t), x i 2(t), \ldots, x i d(t))$,its speed is expressed as $\operatorname{vi}(\mathrm{t})=(\operatorname{vi} 1(\mathrm{t}), \mathrm{vi} 2(\mathrm{t}), \ldots, \operatorname{vid}(\mathrm{t}))$; In the process of iteration, the most optimal position searched by the individual particle can be expressed as $\operatorname{pi}(\mathrm{t})=(\operatorname{pil}(\mathrm{t})$, pi2(t), ...pid(t)), which is called pBest, while the optimal position searched by all particles in the swarm can be presented as $\operatorname{pg}(\mathrm{t})=(\operatorname{pg} 1(\mathrm{t}), \operatorname{pg} 2(\mathrm{t}), \ldots, \operatorname{pgd}(\mathrm{t}))$, named gBest. In each iteration, particles updating these two extremes according to formula (1) and (2) to adjust their flying speed and direction [3]. After $n$ generation of iterations, the most optimal resolution is obtained.

$$
\begin{aligned}
& \operatorname{vid}(t+1)=w^{*} \operatorname{vid}(t)+ \\
& c 1 * r 1 *(\operatorname{pid}(t)-x i d(t)) \\
& +c 2 * r 2 *(\operatorname{pgd}(t)-x i d(t) \\
& \operatorname{xid}(t+1)=\operatorname{xid}(t)+\operatorname{vid}(t+1)
\end{aligned}
$$

In above formulas, $\mathrm{i}=1,2, \ldots, \mathrm{n} ; \mathrm{d}=1,2, \ldots, \mathrm{D} ; \mathrm{r} 1$ and $\mathrm{r} 2$ are symmetrical distribution random numbers between 0 and 1 ; $\mathrm{c} 1$ and $\mathrm{c} 2$ are learning factors representing acceleration constants of the particles flying to the pBest and gBest, usually $\mathrm{c} 1=\mathrm{c} 2=2 ; \mathrm{w}$ is the inertia weight; $\operatorname{vid}(\mathrm{t})$ is the speed of particle $i$ at $t$ moment, $\operatorname{vid}(t+1)$ is the updating speed after iteration. $x i d(t)$ is the position of the particle $i$ at $t$ moment, $\operatorname{xid}(t+1)$ is the updating position after iteration. $\operatorname{pid}(t)$ is the optimal opposition of particle $i$ at $t$-moment, it is the recognition part of the particle; $\operatorname{pgd}(\mathrm{t})$ is the optimal opposition and is the social part of the particle. The first part of the formula (1) shows the global and local search ability of the particle during excise, by introducing inertia weight.

\subsection{ABC Algorithm}

Artificial Bee Colony $(\mathrm{ABC})$ algorithm is a kind of metaheuristic intelligence algorithm and was introduced by Karaboga in 2005 [4]. It was inspired by bees foraging behavior to solve the numerical optimization problem. This method was mainly based on the swarm foraging behavior model proposed by Tereshko and Loengarov in 2005, which 
contains three core elements-- employed bees, unemployed bees and food sources. The first two are responsible for searching rich food sources around hive. This model also defines two kinds of guiding mode, that is to say, a positive signal will be fed back to the swarm by rich food sources which would lead more bees to gather honey. Meanwhile, negative signals will also be fed back result in giving up the food sources [5]. Both behaviors are self-organization and swarm intelligence.

In $\mathrm{ABC}$ algorithm, the solution of problems to be solved is regarded as artificial food. If the food is richer, the quality of the solution is better [6]. Then a flock of artificial bees will search the rich food for a better solution of the relative problem. The coming problem should first be translated into the most optimal solution by using $\mathrm{ABC}$ to find a set of parameter vectors which will make the objective function to be minimum. Artificial bee colony will initialize some solution at random and a better solution is closed by using neighbor search through iteration, thus worse solution is abandoned which improves the quality of solution. In this process, each bee represents a possible solution of the optimal problem. The quality of the nectar source corresponds to the quality of solution which is expressed by fitness [7, 8]. Observing bees will select one of the nectar according to the message shared by honey gathering bees followed by probability formula (3).

$P i=F(\theta i) \sum s p=1 F(\theta p)$

In which, $\theta \mathrm{i}$ is on behalf of the $\mathrm{i}$-th nectar, $\mathrm{i} \in\{1,2, \ldots, \mathrm{S}\}, \mathrm{S}$ represents the number of nectars, $\mathrm{F}(\theta \mathrm{i})$ shows the fitness of the i-th nectar. After comparing to other nectar around $\theta \mathrm{i}$, observing bees will select one of the nectars. Location of the new nectar is calculated as follows:

$\theta_{i}(c+1)=\theta_{i}(c) \pm \varphi_{i}(c)$

In above formula, $\varphi \mathrm{i}(\mathrm{c})$ is the renewal step-size near $\theta \mathrm{i}$ generated randomly. If the fitness of a new nectar is better than the original one, that is, $\mathrm{F}(\theta \mathrm{i}(\mathrm{c}+1))>\mathrm{F}(\theta \mathrm{i}(\mathrm{c}))$, observing bee will select a new nectar $\theta \mathrm{i}(\mathrm{c}+1)$; Otherwise, it stays unchanged. When the number of loops reaches to the limited count, if the quality of nectar gets little improvement, the honey gathering bees will give up this nectar and turn into observing bees and the location of xi will update as follows:

$X i(j)=x \min (j)+\varphi^{\prime}(x \max (j)-x \min (j))$ $[0,1]$.

Here $\varphi^{\prime}$ is a symmetrical distributed random number in

\section{IMPLEMENTATION OF FUSION ALGORITHM}

\subsection{Algorithm Principle}

In our algorithm, $\mathrm{PSO}$ and $\mathrm{ABC}$ algorithms are fused together to form a new PSO-ABC algorithm. At first, the particle swarm is divided into PNum sub-swarms with equal size which will evolve on the basis of PSO. Then the best particles of every sub-swarm constitute a new swarm and pick out the best particle after evolution. Because the position of the best particle of each sub-swarm is changed after evolution, the diversity of population is increased [9]. Meanwhile, PSO algorithm can search precisely to find better solution. That the global best particle's position feedbacks into the speed updating formula can lead the PSO to jump out of local optimum effectively[10]. The speed and Location updating formula of PSO-ABC is described here:

$$
\begin{aligned}
& \operatorname{vid}(t+1)=w^{*} \operatorname{vid}(t)+c 1 * r 1 * \operatorname{pid}(t) \\
& -x i d(t))+c 2 * r 2 * \mu 1 *(\operatorname{pigd}(t)-x i d(t)) \\
& +c 3 * r 3 * \mu 2 *(\operatorname{Xgd}(t)-x i d(t)) \\
& \operatorname{xid}(t+1)=x i d(t)+\operatorname{vid}(t+1)
\end{aligned}
$$

In above formulas, pigd(t) is the global optimal solution of the set of $\mathrm{i}$-th particle swarm at $\mathrm{t}$-moment and $\mathrm{Xgd}(\mathrm{t})$ is the best particle calculated by $\mathrm{ABC}$ algorithm.

Compared with standard particle swarms speed formula, formula (6) expands the third and fourth part with two impact factors- $\mu 1$ and $\mu 2(\mu 1=0.5, \mu 2=0.25)$, which makes the fourth part becoming the global optimal feedback. Particle swarm's speed is controlled at a right level, which decreases the impact on optimal particles, maintains the population's diversity and avoids trapping in local optimization [11].

\subsection{Algorithm Flow}

(1) Initiate the particle swarm and set relative parameters; (2) Divide particles into PNum groups, each has Num particles; (3) Evaluate the fitness of each particle, record optimal particle pigd of each group.; (4) Update the particle swarm according to the formula (6) and (7); (5) Update the global optimal point of each group pigd; (6) Take the global optimal point of each group pigd as the initial particle of $\mathrm{ABC}$ algorithm; (7) Update particles according to the $\mathrm{ABC}$ updating formula to find the optimal point $\mathrm{Xgd}$; (8) If termination condition is satisfied, $\mathrm{Xgd}$ is output and algorithm stops; otherwise returns to (4) step.

\section{SIMULATING EXPERIMENT AND RESULTS ANALYSIS}

To validate the algorithm of this article, the comparison experiment has been processed with the references [1] and [2], implemented in Matlab under the environment of Pentium 2.7GHz CPU and 2GB memory. In this experiment, the problems is transformed into inequality constrained optimization problem, the penalty function is used as benefit function to do iteration. The numerical experiment is done under the condition of gradient linear independence assumption without active constraint and the problem of minimal location is solved.

Assume minimal location problem is as follows: For the $n$ points $\operatorname{Pi}(\mathrm{x}, \mathrm{y})(\mathrm{i}=1,2, \ldots, \mathrm{n})$ in a given plane and the surface area of any shape $R=U j R j$, every $R j$ is continuous region. Note $\partial \mathrm{R}$ as the boundary of $\mathrm{R}$. Depending on the distance measure, Euclidean distance and absolute distance are the two kinds of problems to determine location $\mathrm{P}(\mathrm{x}, \mathrm{y})$ and result in :min $\max \{[\mathrm{x}-\mathrm{xi}] 2+(\mathrm{y}-\mathrm{yi}) 2] 1 / 2 \mid \mathrm{P} \in \mathrm{R} \cup \partial \mathrm{R}\}$.

Example 1 Given 16 points on plane as follows, the results of three kinds of algorithms is shown in Table $\mathbf{1}$.

$\mathrm{P} 1=(-9,8), \mathrm{P} 2=(-15,-8), \mathrm{P} 3=(22,5), \mathrm{P} 4=(17,20), \mathrm{P} 5=(10,0)$, $\mathrm{P} 6=(3,4), \mathrm{P} 7=(-5,-9), \mathrm{P} 8=(-16,-4), \mathrm{P} 9=(12,4), \mathrm{p} 10=(-10,7), \mathrm{P} 11=$ 
Table 1. The results of three kinds of algorithms on example 1.

\begin{tabular}{|c|c|c|c|c|}
\hline \multirow{2}{*}{ Algorithm Name } & \multicolumn{2}{|c|}{ Objective Function Value } & \multicolumn{2}{c|}{ Location Point } \\
\hline \hline & Euclidean Distance & Absolute Distance & Euclidean Distance & Absolute Distance \\
\hline Reference [1] & 52.000 & 57.210 & $(-30.000,5.120)$ & $(-30.150,9.940))$ \\
\hline Reference [2] & 52.000 & 57.110 & $(-30.000,5.100)$ & $(-30.000,10.000)$ \\
\hline This article & 52.000 & 57.000 & $(-30.000,5.000)$ & $(-30.000,9.930)$ \\
\hline
\end{tabular}

Table 2. The results of three kinds of algorithms on example 2.

\begin{tabular}{|c|c|c|c|c|}
\hline \multirow{2}{*}{ Algorithm Name } & \multicolumn{2}{|c|}{ Objective Function Value } & \multicolumn{3}{c|}{ Location Point } \\
\hline & Euclidean Distance & Absolute Distance & Euclidean Distance & Absolute Distance \\
\hline \hline Reference [1] & 10.640 & 14.000 & $(-1.390,1.060)$ & $(-2.300,-0.400))$ \\
\hline Reference [2] & 10.601 & 14.000 & $(-1.300,1.080)$ & $(-1.600,0.500)$ \\
\hline This article & 10.588 & 14.000 & $(-1.311,1.054)$ & $(-1.764,0.365)$ \\
\hline
\end{tabular}

$(1,14), \mathrm{P} 12=(-7,6), \mathrm{P} 13=(-14,3), \mathrm{P} 14=(12,24), \mathrm{P} 15=(-1,1)$, $\mathrm{P} 16=(0,13)$

The composition of region $\mathrm{R}$ is as follows:

$$
\begin{aligned}
& x-y \leq 30 \\
& -2 x-3 y \leq 90 \\
& x \leq-x+4 y \\
& -x+4 y \leq 150 \\
& 7 x+5 y \leq 270 \\
& 11 x-5 y \leq 270
\end{aligned}
$$

Example 2: Given 12 points on plane as follows, the results of three kinds of algorithms is shown in Table 2.

The composition of region $\mathrm{R}$ is as follows:

$$
\begin{aligned}
& 4(x-5 y-30) \leq y 2 \\
& 2 x+3 y \leq 60 \\
& -x+5 y \leq 100 \\
& -9 x+y \leq 240 \\
& -x-4 y \leq 150 \\
& 19 x-24 y \leq 60
\end{aligned}
$$

Seen from Tables $\mathbf{1}$ and $\mathbf{2}$, the results of PSO-ABC have good global search ability and fast convergence speed, which are superior to those of references of [1] and [2] and have the advantages of both PSO and ABC showing a good test effect. When the test results of standard PSO and ABC are not ideal, fusion algorithm can effectively solve the problem. Fusion algorithm can effectively avoid premature convergence and it can approximate gradually to global optimal solution as the search process continues.

\section{CONCLUSION}

Swarm optimization problem, particle swarm and artificial swarm fusion algorithm is proposed as PSO - ABC algo- rithm, and simulation experiments using the minimax location problem's examples show that the results of algorithm proposed in this thesis are better than those of reference [1] and reference [2] algorithm and has good application value.

\section{CONFLICT OF INTEREST}

The authors confirm that this article content has no conflict of interest.

\section{ACKNOWLEDGEMENTS}

This study is supported in part by the National Natural Science Foundation of China (Grant No. 61401242), the Research Foundation for Advanced Talents of Nanyang Normal University (ZX2014058), Technology Research and Development Program of Lianyungang (Grant No. SH1223), the Research plan for Basic and frontier Research of Henan (Grant No. 142300410044), and the Key Programs of Education Department of Henan Province (Grant No. 14A520057).

\section{REFERENCES}

[1] D. G., Xu, and R. B. Xiao, "Application of improved Neural network in grain and oil distribution center location", Computer Engineering and Applications, vol. 45, no. 35, 216-219, 2009.

[2] R. Z. Qiu, X. Y. Zhong, C. Zhong, and X. Lin, "Study on Optimization of Fresh Agricultural Product Distribution Center Location Based on Ant Colony Algorithm", Journal of Jiangnan University (Natural Science Edition), vol. 9, no. 2, 2010.

[3] X. L. Jiang, J. Y. Guo, J. Tang, and H. F., Ling, "Multi-objective Particle Swarm Algorithm based on Fuzzy-learning Sub-swarm", Application Research of Computers. Vol. 28, no. 12, pp. 98-100, 2011.

[4] Z. Y. Lin, and L. L. Wang. "Hybrid artificial bee colony algorithm for solving high-dimensional function optimization problems", Computer Science, vol. 40, no. 3, pp. 279-282, 2013.

[5] L. Dong, and H. W. Xie. "Study on optimization of rank fusion algorithm in metasearch engine", Computer Applications and software, vol. 29, no. 10, pp. 188-190, 2012.

[6] Y. H., LI, X. Y., Zhong, and C. Zhong, "Choice of timber logistics center location based on genetic algorithm", Operations Research and Management Science, vol. 16, no. 6, 2007 (in Chinese). 
[7] L. W. Song, M. F. Peng, C. L. Tian, and S. Meie, "Analog Circuit Diagnosis based on Particle Swarm Optimization Radial Basis Function Network", Application Research of Computers, vol. 29, no. 1, pp. 72-74, 2012.

[8] B. Wei, Y. Li, X. Xu, and D. Shen, "Particle Swarm Optimization Algorithm based on Stable Strategy", Computer Science, vol. 38, no. 12 , pp. 221-223, 2011
[9] L. Huang, W. W. Du, and L. X. Ding, "Adaptive Particle Swarm Optimization Algorithm based on Sigmoid Inertia Weight.", Application Research of Computers, vol. 29, no. 1, pp. 38-40, 2012.

[10] Z. J. Li, "Application of artificial bee colony algorithm in path planning of mobile robot", Computer Simulation, vol. 29 no. 12, pp. 247-250, 2012.

[11] K. Hu, X. B. Li, and Z. L. Wang, "Performance of an improved artificial bee colony algorithm", Journal of Computer Applications, vol. 31, no. 4, pp. 1107-1110, 2011.

Received: September 16, 2014

Revised: December 23, 2014

Accepted: December 31,2014

(C) Wang et al.; Licensee Bentham Open.

This is an open access article licensed under the terms of the (https://creativecommons.org/licenses/by/4.0/legalcode), which permits unrestricted, noncommercial use, distribution and reproduction in any medium, provided the work is properly cited. 